\title{
Human Leukocyte Antigen Associations with Acute Leukemia: An Indian Perspective
}

\begin{abstract}
Objective: Acute lymphoid leukemia (ALL) and acute myeloid leukemia (AML) are neoplastic blood disorders in which the cancerous white blood cells accumulate, resulting in a significant morbidity and mortality. Human leukocyte antigen (HLA) association is observed as one of the factors in the development of leukemia. The objective of the present study was to analyze the allele frequency of HLA Class I (HLA-A, HLA-B, and HLA-C) and Class II (HLA-DRB1 and HLA-DQB1) in Indian acute leukemia patients and to compare them with the frequencies in healthy, unrelated Indian individuals. Materials and Methods: We included 500 Indian leukemic patients $(A M L=324$ and ALL = 176) and 1000 unrelated, healthy, Indian individuals as controls. The HLA typing was performed using polymerase chain reaction with sequence-specific oligonucleotide probes. Results: On univariate analysis, allele frequencies of HLA-A*11 and HLA-DRB1*11 were lower in patients with ALL $(P=0.0181$ and $P=0.0025$, respectively). Whereas of HLA-A*11, HLA-DRB1*11, and HLA-B*51, these frequencies were relatively lower in patients with acute leukemia (AML $+\operatorname{ALL})(P=0.0382, P=0.0093$ and $P=0.0384$, respectively) and HLA-C*01 $(P=0.0304)$ in AML when compared with control individuals. In contrast, the HLA-B*39 and HLA-C*07 allele frequency was higher in acute leukemia $(P=0.00372$ and $P=0.0463$, respectively) and in AML $(P=0.0010$ and $P=0.0178$, respectively) than that in controls. On multivariate analysis, $\mathrm{B} * 39$ showed positive associations with acute leukemia $(P=0.006)$ and AML $(P=0.002)$. HLA-A*11 and-DRB $1 * 11$ showed a negative association with acute leukemia $(P=0.009$ and $P<0.0001$, respectively) and ALL $(P=0.013$ and $P<0.0001$, respectively). Conclusions: The HLA-B*39 has a positive association with AML and acute leukemia, whereas HLA-A*11 and HLA-DRB1*11 alleles have negative association with ALL and HLA-B*51 along with these two alleles with acute leukemia. No positive association was observed with ALL. HLA-C*01 frequency was lower in AML patients than that in controls.
\end{abstract}

Keywords: Acute lymphoid leukemia, acute myeloid leukemia, alleles, human leukocyte antigen, leukemia, polymerase chain reaction with sequence-specific oligonucleotide probes

\section{Introduction}

Leukemia is a type of cancer that targets the leukocytes. The cell lineage affected by the cancer determines the kind of leukemia and the affect can be sudden or "acute" or can develop slowly or "chronic." $[1]$ Acute myeloid leukemia (AML) is a malignant neoplasm of myeloid cells originating in and infiltrating the bone marrow. Acute lymphoid leukemia (ALL) originates in lymphoid cells. ${ }^{[2]}$

The human major histocompatibility complex (MHC) is called the human leukocyte antigen (HLA) system as these molecules can be detected on the surface of leukocytes using specific alloantibodies. ${ }^{[3]}$ The HLA complex of genes is found on the short arm of chromosome 6 . They play an

This is an open access journal, and articles are distributed under the terms of the Creative Commons Attribution-NonCommercial-ShareAlike 4.0 License, which allows others to remix, tweak, and build upon the work non-commercially, as long as appropriate credit is given and the new creations are licensed under the identical terms.

For reprints contact: WKHLRPMedknow_reprints@wolterskluwer.com important role in incompatibility matching between donor and recipient for transplantations. ${ }^{[4]}$ The HLA loci include Class I (HLA-A, -B, and -C) and Class II (HLA-DR, -DQ, and -DP) antigens. The HLA genes are the most commonly known polymorphic genes. The frequencies of HLA antigens differ among different populations. ${ }^{[5]}$ The HLA has a property of nonrandom association of HLA alleles across the genome, which is known as linkage disequilibrium. ${ }^{[6]}$ The role of MHC and its associations have been studied in over fifty different diseases, and many of them have viral origination. ${ }^{[7]}$

The first MHC association in mouse leukemia was reported by Lilly in 1964; since then, the studies on HLA association with leukemia began. ${ }^{[8]}$ In 1967, the first HLA study in ALL was reported with an increased frequency of HLA-A2. ${ }^{[9]}$ In

How to cite this article: Solanki H, Mishra VC,
Tiwari AK, Kakkar N, Vashisht N, Raina V, et al.
Human leukocyte antigen associations with acute
leukemia: An Indian perspective. Indian J Med
Paediatr Oncol 2020;41:850-8.

\section{Hina Solanki ${ }^{1,2}$, Vikash C. Mishra ${ }^{1,2}$, Aseem K. Tiwari ${ }^{3}$, Nipun Kakkar ${ }^{4}$, Naveen Vashisht ${ }^{1}$, Vimarsh Raina ${ }^{1}$, Girish Sharma ${ }^{2,5}$}

${ }^{1}$ Chimera Transplant Research Foundation, Masjid Moth, South Extension, New Delhi, India, ${ }^{2}$ Centre for Medical Biotechnology, Amity Institute of Biotechnology, Amity University, Noida, Uttar Pradesh, India. ${ }^{3}$ Department of Transfusion Medicine, Medanta The Medicity, Gurgaon, Haryana, India, ${ }^{4}$ Department of Pediatric Oncology, Baylor College of Medicine, Houston, Texas, USA, ${ }^{5}$ Amity Center for Cancer Epidemiology and Cancer Research, Amity University, Noida, Uttar Pradesh, India

Submitted: 26-Apr-2020

Revised: 12-Aug-2020

Accepted: 02-Oct-2020

Published: 31-Dec-2020

Address for correspondence:

Dr. Girish Sharma,

Amity Center for Cancer Epidemiology and Cancer Research, Amity University, Sector-125, Noida, Uttar Pradesh, India. E-mail: sharmagi03@gmail. com; gsharma3@amity.edu

Access this article online

Website: www.ijmpo.org

DOI: 10.4103/ijmpo.ijmpo_195_20 Quick Response Code:

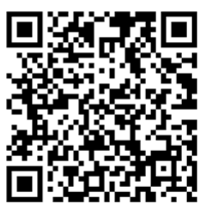


1970, the first HLA haplotype association in ALL was reported with HLA-A2B12. ${ }^{[10]}$ The association of HLA-A2 is still not confirmed in any of the studies. Seremetis et al. reported one of the most powerful associations on AML patients examining DRB loci. ${ }^{[11]}$ The largest HLA association study reported in 1987, done on International Bone Marrow Transplant Registry data, showed HLA-Cw3 and $\mathrm{Cw} 4$ associations with AML and ALL. ${ }^{[12]}$ Another study in 1979 showed HLA-Cw7 association with ALL. ${ }^{[13]}$ All these studies done to find the HLA disease association were serological ones. A recent study done on Korean population claimed the HLA-C3 association with AML. ${ }^{[14]}$ As stated earlier, HLA is highly polymorphic and its distribution varies with population. The HLA associations with acute leukemia have not been studied in Indian patients, so it is important to study this disease in the Indian population. The objective of the present study was to analyze the allele frequency of HLA Class I (HLA-A, HLA-B, and HLA-C) and Class II (HLA-DRB1 and HLA-DQB1) in Indian acute leukemia patients. This is possibly the first HLA leukemia association study in the Indian population.

\section{Materials and Methods}

\section{Patients}

This prospective observational study was carried out at a clinical laboratory in the national capital region of India. The study included data collected from patients diagnosed with ALL and acute AML (no further classification on AML and ALL was studied), who visited the clinical laboratory during the study period of January 2013 to December 2019 for the tests and mostly were BMT (bone marrow transplant) candidates. The data were collected from 500 Indian acute leukemia patients (males $=328$, females $=172$ ) regarding their age, gender, year of diagnosis, type of disease, and laboratory reports such as peripheral blood counts and medical history. Out of the total 500 samples, AML samples were of 304 (males = 203, females $=121$ ) and ALL samples collected were of 176 (males $=125$, females $=51)$. It was ensured that the patients are not repeated in case they have visited the laboratory more than once during the study period. The study was approved by the Institutional Ethics Committee for Health Related Research.

\section{Control group}

The control group consisted of 1000 (males $=663$, females $=337$ ) healthy unrelated donors and volunteers from a bone marrow transplant registry (Genebandhu), ${ }^{[15]}$ which is also located in the national capital region of India. All the individuals were of Indian origin. The controls were age- and gender-matched to the patient groups.

\section{Human leukocyte antigen typing}

The HLA tissue typing was performed at the clinical laboratory situated in the national capital region. The laboratory has ISO15189 accreditation to carry out HLA typing from the National Accreditation Board of Testing and Calibration Laboratories (NABL). DNA was extracted by Nucleospin $^{R}$ DNA extraction kit (MACHEREY-NAGEL, $\mathrm{GmbH}$ and Co. KG, Düren, Germany). HLA-A, -B, -C and -DRB1 and -DQB1 typing was performed with polymerase chain reaction with sequence-specific oligonucleotide probe (PCR-SSOP) technique using Lifecodes HLA SSO typing kits (Immucor, Stanford, CT, USA) on Luminex platform.

\section{Statistical analysis}

The allele frequencies were calculated by direct count using Microsoft Excel 2007. Comparison between the two observed frequencies was made using Chi-square analysis including Yates correction where the cell frequency was $<20$. When the observed frequency was $<5$, Fisher's exact test was performed. $P \leq 0.05$ was considered statistically significant. An odds ratio (OR) with a 95\% confidence interval (CI) was employed for risk estimation using Epilnfo statistical program (version 6, Centers for Disease Control and Prevention, Atlanta, GA, USA). Multivariate logistic regression was performed and presented as $\mathrm{OR}$ and $95 \% \mathrm{CI}, P \leq 0.05$ was considered statistically significant. Statistical analysis was performed using SPSS software (version 24.0; IBM Corp, Bengaluru, Karnataka, India).

\section{Results}

A total of 500 leukemic patients were studied; all the 500 patients were tested on HLA-A, -B, and -DRB1 loci and 470 were tested on $-\mathrm{C}$ and -DQB1 loci. In the AML group, a total of 324 patients were studied; all the 324 patients were tested on HLA-A, -B, and -DR loci and 306 were tested on $-\mathrm{C}$ and -DQB1 loci. In the ALL group, a total of 176 patients were studied and all the 176 patients were tested on HLA-A, -B, and -DR loci and 164 were tested on $-\mathrm{C}$ and $-\mathrm{DQB} 1$ loci. A total of 1000 unrelated healthy individuals as controls were studied at HLA-A, -B, -C, -DRB1, and -DQB1 loci. The allele frequencies were calculated instead of gene frequencies. ${ }^{[16]}$

Distribution of human leukocyte antigen frequencies between acute leukemia patients and controls

The frequency of HLA-A, -B, -C, -DRB1, and -DQB1 alleles in patients with acute leukemia and control is summarized in Tables 1 and 2. Significant differences were observed in the allelic distribution of patients with acute leukemia compared vis a vis controls upon studying HLA Class I (-A, -B, and -C) and HLA Class II (-DRB1 and $-\mathrm{DQB} 1)$ loci. A significant negative association of the HLA-A*11, HLA-B*51, and HLA-DRB1*11 alleles with acute leukemia $[26.4 \%$ vs. $31.6 \%, P=0.0382 ; 13.6 \%$ vs. $17.8 \%, P=0.0384$; and $16.2 \%$ vs. $21.9 \%, P=0.0093$, respectively; Tables 1 and 2] was observed. In contrast, the frequency of HLA-B*39 and HLA-C*07 allele was 
Table 1: Human leukocyte antigen-A, -B, -DR allele frequencies in Indian acute leukemia patients and controls

\begin{tabular}{|c|c|c|c|c|c|c|}
\hline \multirow{2}{*}{$\begin{array}{l}\text { HLA } \\
\text { Allele }\end{array}$} & \multirow{2}{*}{$\begin{array}{l}\text { Frequencies }(\%) \text { in acute } \\
\text { leukemic patients }(n=500)\end{array}$} & \multirow{2}{*}{$\begin{array}{l}\text { Frequencies }(\%) \text { in } \\
\text { control }(n=1000)\end{array}$} & \multirow[t]{2}{*}{$P$} & \multirow[t]{2}{*}{ OR } & \multicolumn{2}{|c|}{$95 \% \mathrm{CI}$} \\
\hline & & & & & Low & High \\
\hline $\mathrm{A}^{*} 01$ & 24 & 23.5 & NS & & & \\
\hline $\mathrm{A} * 02$ & 29.4 & 29.1 & NS & & & \\
\hline$A * 03$ & 18.6 & 15.4 & NS & & & \\
\hline$A^{*} 11$ & 26.4 & 31.6 & 0.0382 & 0.7764 & 0.6111 & 0.9864 \\
\hline$A * 23$ & 2.6 & 2.3 & NS & & & \\
\hline$A * 24$ & 26.4 & 27.1 & NS & & & \\
\hline$A * 25$ & 0.2 & 0 & NS & & & \\
\hline$A * 26$ & 9.2 & 12.3 & NS & & & \\
\hline$A * 29$ & 1.4 & 2 & NS & & & \\
\hline$A * 30$ & 3.6 & 2.9 & NS & & & \\
\hline$A * 31$ & 5.4 & 6.1 & NS & & & \\
\hline$A * 32$ & 7.2 & 8.5 & NS & & & \\
\hline$A * 33$ & 16.8 & 14 & NS & & & \\
\hline$A * 34$ & 0.2 & 0 & NS & & & \\
\hline$A * 68$ & 12.6 & 11.7 & NS & & & \\
\hline A*74 & 0.8 & 0.2 & NS & & & \\
\hline $\mathrm{B} * 03$ & 0.4 & 0 & NS & & & \\
\hline $\mathrm{B} * 07$ & 12.2 & 10.4 & NS & & & \\
\hline $\mathrm{B} * 08$ & 10.2 & 13.3 & NS & & & \\
\hline $\mathrm{B} * 13$ & 4.8 & 4.5 & NS & & & \\
\hline B*14 & 0.6 & 0.2 & NS & & & \\
\hline $\mathrm{B} * 15$ & 18.4 & 16 & NS & & & \\
\hline B*18 & 7.4 & 5.9 & NS & & & \\
\hline$B * 27$ & 4.2 & 4 & NS & & & \\
\hline$B * 35$ & 24.4 & 29.1 & NS & & & \\
\hline$B * 37$ & 4.6 & 5.3 & NS & & & \\
\hline$B * 38$ & 3.2 & 2 & NS & & & \\
\hline$B * 39$ & 1.8 & 0.3 & 0.00372 & 6.0916 & 1.6418 & 22.6022 \\
\hline$B * 40$ & 17.8 & 21.7 & NS & & & \\
\hline$B * 41$ & 1.6 & 1.6 & NS & & & \\
\hline$B * 42$ & 0.2 & 0 & NS & & & \\
\hline$B * 43$ & 0.2 & 0 & NS & & & \\
\hline$B * 44$ & 17.6 & 14.9 & NS & & & \\
\hline$B * 45$ & 0.2 & 0.2 & NS & & & \\
\hline$B * 47$ & 0.4 & 0.5 & NS & & & \\
\hline$B * 48$ & 1.6 & 1.8 & NS & & & \\
\hline$B * 49$ & 1.8 & 0.7 & NS & & & \\
\hline $\mathrm{B} * 50$ & 5.6 & 5.4 & NS & & & \\
\hline$B * 51$ & 13.6 & 17.8 & 0.0384 & 0.7269 & 0.537 & 0.9839 \\
\hline$B * 52$ & 15.8 & 15.2 & NS & & & \\
\hline$B * 53$ & 0.4 & 0.3 & NS & & & \\
\hline$B * 55$ & 3.6 & 4.2 & NS & & & \\
\hline$B * 56$ & 1 & 1.4 & NS & & & \\
\hline$B * 57$ & 7.8 & 6.8 & NS & & & \\
\hline$B * 58$ & 8.6 & 7.4 & NS & & & \\
\hline $\mathrm{DRB} 1 * 01$ & 7.2 & 4.9 & NS & & & \\
\hline $\mathrm{DRB} 1 * 02$ & 0.4 & 0 & NS & & & \\
\hline $\mathrm{DRB} 1 * 03$ & 20.2 & 23.4 & NS & & & \\
\hline $\mathrm{DRB} 1 * 04$ & 14.4 & 14 & NS & & & \\
\hline $\mathrm{DRB} 1 * 06$ & 0.2 & 0.1 & NS & & & \\
\hline $\mathrm{DRB} 1 * 07$ & 30 & 26.6 & NS & & & \\
\hline $\mathrm{DRB} 1 * 08$ & 2.4 & 3.8 & NS & & & \\
\hline
\end{tabular}




\begin{tabular}{|c|c|c|c|c|c|c|}
\hline \multicolumn{7}{|c|}{ Table 1: Contd... } \\
\hline \multirow{2}{*}{$\begin{array}{l}\text { HLA } \\
\text { Allele }\end{array}$} & \multirow{2}{*}{$\begin{array}{l}\text { Frequencies }(\%) \text { in acute } \\
\text { leukemic patients }(n=500)\end{array}$} & \multirow{2}{*}{$\begin{array}{l}\text { Frequencies }(\%) \text { in } \\
\text { control }(n=1000)\end{array}$} & \multirow[t]{2}{*}{$P$} & \multirow[t]{2}{*}{ OR } & \multicolumn{2}{|c|}{$95 \% \mathrm{CI}$} \\
\hline & & & & & Low & High \\
\hline$\overline{\mathrm{DRB} 1 * 09}$ & 2.2 & 1.3 & NS & & & \\
\hline $\mathrm{DRB} 1 * 10$ & 8.6 & 10 & NS & & & \\
\hline $\mathrm{DRB} 1 * 11$ & 16.2 & 21.9 & 0.0093 & 0.6894 & 0.5204 & 0.9132 \\
\hline $\mathrm{DRB} 1 * 12$ & 5.6 & 4.4 & NS & & & \\
\hline $\mathrm{DRB} 1 * 13$ & 19 & 20.9 & NS & & & \\
\hline $\mathrm{DRB} 1 * 14$ & 16.8 & 16.1 & NS & & & \\
\hline $\mathrm{DRB} 1 * 15$ & 35 & 35.1 & NS & & & \\
\hline $\mathrm{DRB} 1 * 16$ & 1.6 & 2.2 & NS & & & \\
\hline $\mathrm{DRB} 1 * 17$ & 0.2 & 0 & NS & & & \\
\hline
\end{tabular}

NS - Not significant; HLA - Human leukocyte antigen; OR - Odds ratio; CI - Confidence interval

Table 2: Human leukocyte antigen-C and -DQB1 allele frequencies in Indian acute leukemia patients and controls

\begin{tabular}{|c|c|c|c|c|c|c|}
\hline \multirow[t]{2}{*}{ HLA allele } & \multirow{2}{*}{$\begin{array}{l}\text { Frequencies }(\%) \text { in acute } \\
\text { leukemic patients }(n=470)\end{array}$} & \multirow{2}{*}{$\begin{array}{l}\text { Frequencies }(\%) \text { in } \\
\text { control }(n=1000)\end{array}$} & \multirow[t]{2}{*}{$P$} & \multirow[t]{2}{*}{ OR } & \multicolumn{2}{|c|}{$95 \% \mathrm{CI}$} \\
\hline & & & & & Low & High \\
\hline $\mathrm{C}^{*} 01$ & 5.74 & 7.7 & NS & & & \\
\hline$C^{*} 02$ & 2.77 & 2.6 & NS & & & \\
\hline$C^{*} 03$ & 14.68 & 13.6 & NS & & & \\
\hline$C^{*} 04$ & 26.17 & 28.1 & NS & & & \\
\hline$C^{*} 05$ & 1.91 & 1.9 & NS & & & \\
\hline$C^{*} 06$ & 20.85 & 19.9 & NS & & & \\
\hline $\mathrm{C}^{*} 07$ & 49.15 & 43.6 & 0.0463 & 1.2503 & 1.0035 & 1.5577 \\
\hline$C^{*} 08$ & 3.83 & 4.7 & NS & & & \\
\hline$C^{*} 12$ & 26.60 & 29.2 & NS & & & \\
\hline$C^{*} 14$ & 4.04 & 6 & NS & & & \\
\hline$C^{*} 15$ & 18.09 & 21 & NS & & & \\
\hline$C^{*} 16$ & 6.60 & 4.5 & NS & & & \\
\hline$C^{*} 17$ & 1.49 & 1.4 & NS & & & \\
\hline$C^{*} 18$ & 0.21 & 0 & NS & & & \\
\hline DQB $1 * 01$ & 0.21 & 0 & NS & & & \\
\hline DQB1*02 & 41.70 & 41.5 & NS & & & \\
\hline DQB $1 * 03$ & 41.70 & 45.1 & NS & & & \\
\hline DQB1*04 & 2.34 & 3.8 & NS & & & \\
\hline DQB $1 * 05$ & 39.57 & 36.9 & NS & & & \\
\hline DQB1*06 & 47.02 & 45.3 & NS & & & \\
\hline DQB1*07 & 0.21 & 0 & NS & & & \\
\hline DQB1*15 & 0.21 & 0 & NS & & & \\
\hline
\end{tabular}

NS - Not significant; HLA - Human leukocyte antigen; OR - Odds ratio; CI - Confidence interval

significantly higher in patients than that in controls $(1.8 \%$ vs. $0.3 \%, P=0.00372: 49.15 \%$ vs. $43.6 \%, P=0.0463)$.

Distribution of human leukocyte antigen frequencies between acute myeloid leukemia patients and controls

The frequency of HLA alleles in AML patients and controls is summarized in Tables 3 and 4 . The frequencies of HLA-B*39 $(1.69 \%$ vs. $0.3 \%, P=0.0010)$ and HLA-C*07 (51.31\% vs. $43.6 \%, P=0.0178)$ were higher in AML patients as compared to that of the controls. HLA-C*01 was found to have negative association with AML (3.92\% vs. $7.7 \%, P=0.0304)$.
Distribution of human leukocyte antigen frequencies between acute lymphoid leukemia patients and controls

The HLA frequency distribution in ALL patients and controls is summarized in Tables 5 and 6. The frequencies of HLA-A*11 $(22.73 \%$ vs. $31.6 \%, \quad P=0.0181)$ and $-\mathrm{DRB} 1 * 11(11.93 \%$ vs. $21.9 \%, P=0.0025)$ in ALL patients were decreasing than that in controls. No positive associations were observed with ALL in this study.

\section{Multivariate analysis}

The multivariate analysis results are shown in Table 7. The multivariate analysis showed that $A^{*} 11(\mathrm{OR}=0.719$, 


\begin{tabular}{|c|c|c|c|c|c|c|}
\hline \multirow[t]{2}{*}{ HLA allele } & \multirow{2}{*}{$\begin{array}{c}\begin{array}{c}\text { Frequencies (\%) in AML } \\
(n=324)\end{array}\end{array}$} & \multirow{2}{*}{$\begin{array}{l}\text { Frequencies (\%) in control } \\
\qquad(n=1000)\end{array}$} & \multirow[t]{2}{*}{$P$} & \multirow[t]{2}{*}{ OR } & \multicolumn{2}{|c|}{$95 \%$ CI } \\
\hline & & & & & Low & High \\
\hline$\overline{A * 01}$ & 15.61 & 23.5 & NS & & & \\
\hline$A * 02$ & 20.46 & 29.1 & NS & & & \\
\hline$A * 03$ & 11.81 & 15.4 & NS & & & \\
\hline$A * 11$ & 19.41 & 31.6 & NS & & & \\
\hline$A * 23$ & 2.32 & 2.3 & NS & & & \\
\hline$A * 24$ & 18.14 & 27.1 & NS & & & \\
\hline$A * 25$ & 0.21 & 0 & NS & & & \\
\hline$A * 26$ & 6.12 & 12.3 & NS & & & \\
\hline$A * 29$ & 1.05 & 2 & NS & & & \\
\hline$A * 30$ & 3.16 & 2.9 & NS & & & \\
\hline$A * 31$ & 2.95 & 6.1 & NS & & & \\
\hline$A * 32$ & 3.59 & 8.5 & NS & & & \\
\hline$A * 33$ & 11.81 & 14 & NS & & & \\
\hline$A * 68$ & 8.017 & 11.7 & NS & & & \\
\hline$A * 74$ & 0.63 & 0.2 & NS & & & \\
\hline B*03 & 0.42 & 0 & NS & & & \\
\hline B*07 & 8.017 & 10.4 & NS & & & \\
\hline B*08 & 7.38 & 13.3 & NS & & & \\
\hline B*13 & 4.22 & 4.5 & NS & & & \\
\hline B*14 & 0.42 & 0.2 & NS & & & \\
\hline$B * 15$ & 12.24 & 16 & NS & & & \\
\hline B*18 & 5.49 & 5.9 & NS & & & \\
\hline$B * 27$ & 2.95 & 4 & NS & & & \\
\hline B*35 & 17.30 & 29.1 & NS & & & \\
\hline B*37 & 2.74 & 5.3 & NS & & & \\
\hline$B * 38$ & 1.90 & 2 & NS & & & \\
\hline$B * 39$ & 1.69 & 0.3 & 0.0010 & 8.4135 & 2.2186 & 31.9057 \\
\hline$B * 40$ & 12.02 & 21.7 & NS & & & \\
\hline$B * 41$ & 1.05 & 1.6 & NS & & & \\
\hline$B * 42$ & 0.21 & 0 & NS & & & \\
\hline$B * 44$ & 11.18 & 14.9 & NS & & & \\
\hline$B * 47$ & 0.42 & 0.5 & NS & & & \\
\hline$B * 48$ & 1.48 & 1.8 & NS & & & \\
\hline$B * 49$ & 1.48 & 0.7 & NS & & & \\
\hline$B * 50$ & 3.38 & 5.4 & NS & & & \\
\hline$B * 51$ & 9.28 & 17.8 & NS & & & \\
\hline$B * 52$ & 10.76 & 15.2 & NS & & & \\
\hline$B * 53$ & 0.21 & 0.3 & NS & & & \\
\hline$B * 55$ & 1.69 & 4.2 & NS & & & \\
\hline$B * 56$ & 0.63 & 1.4 & NS & & & \\
\hline$B * 57$ & 4.85 & 6.8 & NS & & & \\
\hline B*58 & 6.54 & 7.4 & NS & & & \\
\hline DRB $1 * 01$ & 4.43 & 4.9 & NS & & & \\
\hline DRB $1 * 02$ & 0.42 & 0 & NS & & & \\
\hline DRB $1 * 03$ & 14.14 & 23.4 & NS & & & \\
\hline DRB $1 * 04$ & 9.70 & 14 & NS & & & \\
\hline DRB $1 * 07$ & 20.89 & 26.6 & NS & & & \\
\hline DRB $1 * 08$ & 1.48 & 3.8 & NS & & & \\
\hline DRB $1 * 09$ & 1.69 & 1.3 & NS & & & \\
\hline $\mathrm{DRB} 1 * 10$ & 5.91 & 10 & NS & & & \\
\hline DRB1*11 & 12.66 & 21.9 & NS & & & \\
\hline
\end{tabular}




\begin{tabular}{|c|c|c|c|c|c|c|}
\hline \multicolumn{7}{|c|}{ Table 3: Contd... } \\
\hline \multirow[t]{2}{*}{ HLA allele } & \multirow{2}{*}{$\begin{array}{l}\text { Frequencies (\%) in AML } \\
(n=324)\end{array}$} & \multirow{2}{*}{$\begin{array}{l}\text { Frequencies }(\%) \text { in control } \\
\qquad(n=1000)\end{array}$} & \multirow[t]{2}{*}{$P$} & \multirow[t]{2}{*}{ OR } & \multicolumn{2}{|c|}{$95 \%$ CI } \\
\hline & & & & & Low & High \\
\hline$\overline{\mathrm{DRB} 1} * 12$ & 2.95 & 4.4 & NS & & & \\
\hline $\mathrm{DRB} 1 * 13$ & 11.81 & 20.9 & NS & & & \\
\hline $\mathrm{DRB} 1 * 14$ & 12.45 & 16.1 & NS & & & \\
\hline $\mathrm{DRB} 1 * 15$ & 23.21 & 35.1 & NS & & & \\
\hline $\mathrm{DRB} 1 * 16$ & 1.27 & 2.2 & NS & & & \\
\hline
\end{tabular}

NS - Not significant; AML - Acute myeloid leukemia; HLA - Human leukocyte antigen; OR - Odds ratio; CI - Confidence interval

\begin{tabular}{|c|c|c|c|c|c|c|}
\hline \multirow[t]{2}{*}{ HLA allele } & \multirow{2}{*}{$\begin{array}{c}\text { Frequencies (\%) in AML } \\
(n=306)\end{array}$} & \multirow{2}{*}{$\begin{array}{l}\text { Frequencies }(\%) \text { in control } \\
\qquad(n=1000)\end{array}$} & \multirow[t]{2}{*}{$P$} & \multirow[t]{2}{*}{ OR } & \multicolumn{2}{|c|}{$95 \%$ CI } \\
\hline & & & & & Low & High \\
\hline $\mathrm{C}^{*} 01$ & 3.92 & 7.7 & 0.0304 & 0.4893 & 0.2626 & 0.9116 \\
\hline$C^{*} 02$ & 2.61 & 2.6 & NS & & & \\
\hline $\mathrm{C}^{*} 03$ & 15.03 & 13.6 & NS & & & \\
\hline$C^{*} 04$ & 27.12 & 28.1 & NS & & & \\
\hline$C^{*} 05$ & 0.98 & 1.9 & NS & & & \\
\hline$C^{*} 06$ & 20.26 & 19.9 & NS & & & \\
\hline $\mathrm{C}^{*} 07$ & 51.31 & 43.6 & 0.0178 & 1.363 & 1.0545 & 1.7619 \\
\hline$C^{*} 08$ & 3.92 & 4.7 & NS & & & \\
\hline$C^{*} 12$ & 25.49 & 29.2 & NS & & & \\
\hline$C^{*} 14$ & 4.58 & 6 & NS & & & \\
\hline$C^{*} 15$ & 19.28 & 21 & NS & & & \\
\hline$C^{*} 16$ & 6.21 & 4.5 & NS & & & \\
\hline$C^{*} 17$ & 1.31 & 1.4 & NS & & & \\
\hline DQB $1 * 01$ & 0.33 & 0 & NS & & & \\
\hline $\mathrm{DQB} 1 * 02$ & 40.85 & 41.5 & NS & & & \\
\hline DQB $1 * 03$ & 42.48 & 45.1 & NS & & & \\
\hline DQB $1 * 04$ & 2.29 & 3.8 & NS & & & \\
\hline $\mathrm{DQB} 1 * 05$ & 40.20 & 36.9 & NS & & & \\
\hline $\mathrm{DQB} 1 * 06$ & 46.73 & 45.3 & NS & & & \\
\hline $\mathrm{DQB} 1 * 07$ & 0.33 & 0 & NS & & & \\
\hline
\end{tabular}

NS - Not significant; AML - Acute myeloid leukemia; HLA - Human leukocyte antigen; OR - Odds ratio; CI - Confidence interval

$\mathrm{CI}=0.561-0.922, P=0.009), \quad \mathrm{B} * 51 \quad(\mathrm{OR}=0.697$, $\mathrm{CI}=0.509-0.955, P=0.025)$, and $\mathrm{DRB} 1 * 11(\mathrm{OR}=3.949$, $\mathrm{CI}=2.705-5.764, P<0.0001)$ were negatively associated with acute leukemia, whereas $\mathrm{B} * 39(\mathrm{OR}=6.629$, $\mathrm{CI}=1.725-25.471, P=0.006)$ showed positive associations with acute leukemia.

With AML, HLA-B*39 $(\mathrm{OR}=8.107, \mathrm{CI}=2.135-30.781$, $P=0.002)$ showed a positive association whereas HLA-C*01 showed a negative association $(\mathrm{OR}=0.489$, $\mathrm{CI}=0.262-0.912, P=0.025)$.

With ALL, HLA-A $* 11(\mathrm{OR}=0.617, \mathrm{CI}=0.422-0.902$, $P=0.013)$ and $-\mathrm{DRB} 1 * 11(\mathrm{OR}=2.740, \mathrm{CI}=1.593-4.714$, $P<0.0001)$ showed significance and negative association.

\section{Discussion}

The studies on HLA and its association with different diseases began soon after the standardization of serology techniques. The first study on HLA association with leukemia was done in 1967, which showed that the frequency of HLA-A2 in ALL patients was higher. ${ }^{[9]}$ It is important to study the HLA genes and acute leukemia as these genes may have positive or negative associations in the development of leukemia. ${ }^{[17]}$

In our study, no positive association was observed between HLA alleles and ALL using the PCR-SSOP technique, which is matching with that reported by Villalobos et al. who worked with the PCR-sequence-specific primer and sequence-specific oligonucleotide techniques on Venezuelan population. ${ }^{[18]}$

In the present study, no increased frequency of any individual HLA-A, HLA-DRB1, and HLA-DQB1 antigen was observed in patients with acute leukemia, AML, or in ALL compared with controls, but the frequency of HLA-A*11 and HLA-DRB1*11 was lower in ALL and in acute leukemia as compared with the controls, suggesting their negative association with the disease. Earlier, in a study on Turkish population, the frequency of HLA-A*11 in AML patients was observed to be decreasing. ${ }^{[19]}$ 


\begin{tabular}{|c|c|c|c|c|c|c|}
\hline \multirow[t]{2}{*}{ HLA allele } & \multirow{2}{*}{$\begin{array}{c}\text { Frequencies (\%) in ALL } \\
(n=176)\end{array}$} & \multirow{2}{*}{$\begin{array}{l}\text { Frequencies (\%) in control } \\
\qquad(n=1000)\end{array}$} & \multirow[t]{2}{*}{$P$} & \multirow[t]{2}{*}{ OR } & \multicolumn{2}{|c|}{$95 \%$ CI } \\
\hline & & & & & Low & High \\
\hline $\mathrm{A}^{*} 01$ & 26.14 & 23.5 & NS & & & \\
\hline $\mathrm{A} * 02$ & 28.41 & 29.1 & NS & & & \\
\hline$A * 03$ & 21.02 & 15.4 & NS & & & \\
\hline A*11 & 22.73 & 31.6 & 0.0181 & 0.6366 & 0.4367 & 0.9281 \\
\hline$A * 23$ & 1.14 & 2.3 & NS & & & \\
\hline$A * 24$ & 26.14 & 27.1 & NS & & & \\
\hline$A * 26$ & 9.66 & 12.3 & NS & & & \\
\hline$A * 29$ & 1.14 & 2 & NS & & & \\
\hline$A * 30$ & 1.70 & 2.9 & NS & & & \\
\hline$A * 31$ & 7.39 & 6.1 & NS & & & \\
\hline$A * 32$ & 10.80 & 8.5 & NS & & & \\
\hline$A * 33$ & 15.91 & 14 & NS & & & \\
\hline$A * 34$ & 0.57 & 0 & NS & & & \\
\hline$A * 68$ & 14.20 & 11.7 & NS & & & \\
\hline A*74 & 0.57 & 0.2 & NS & & & \\
\hline $\mathrm{B} * 07$ & 13.07 & 10.4 & NS & & & \\
\hline $\mathrm{B} * 08$ & 9.09 & 13.3 & NS & & & \\
\hline$B * 13$ & 2.27 & 4.5 & NS & & & \\
\hline$B * 14$ & 0.57 & 0.2 & NS & & & \\
\hline$B * 15$ & 19.32 & 16 & NS & & & \\
\hline B*18 & 6.25 & 5.9 & NS & & & \\
\hline$B * 27$ & 3.98 & 4 & NS & & & \\
\hline B*35 & 22.73 & 29.1 & NS & & & \\
\hline$B * 37$ & 5.68 & 5.3 & NS & & & \\
\hline B*38 & 3.98 & 2 & NS & & & \\
\hline$B * 39$ & 0.57 & 0.3 & NS & & & \\
\hline$B * 40$ & 18.18 & 21.7 & NS & & & \\
\hline$B * 41$ & 1.70 & 1.6 & NS & & & \\
\hline$B * 43$ & 0.57 & 0 & NS & & & \\
\hline$B * 44$ & 19.89 & 14.9 & NS & & & \\
\hline$B * 45$ & 0.57 & 0.2 & NS & & & \\
\hline$B * 48$ & 0.57 & 1.8 & NS & & & \\
\hline$B * 49$ & 1.14 & 0.7 & NS & & & \\
\hline B*50 & 6.82 & 5.4 & NS & & & \\
\hline$B * 51$ & 13.64 & 17.8 & NS & & & \\
\hline$B * 52$ & 15.91 & 15.2 & NS & & & \\
\hline $\mathrm{B} * 53$ & 0.57 & 0.3 & NS & & & \\
\hline $\mathrm{B} * 55$ & 5.68 & 4.2 & NS & & & \\
\hline$B * 56$ & 1.14 & 1.4 & NS & & & \\
\hline$B * 57$ & 9.09 & 6.8 & NS & & & \\
\hline B*58 & 6.82 & 7.4 & NS & & & \\
\hline DRB1*01 & 8.52 & 4.9 & NS & & & \\
\hline DRB1*03 & 19.32 & 23.4 & NS & & & \\
\hline DRB1*04 & 14.77 & 14 & NS & & & \\
\hline DRB1*06 & 0.57 & 0.1 & NS & & & \\
\hline DRB1*07 & 28.98 & 26.6 & NS & & & \\
\hline DRB $1 * 08$ & 2.84 & 3.8 & NS & & & \\
\hline DRB1*09 & 1.70 & 1.3 & NS & & & \\
\hline DRB1*10 & 8.52 & 10 & NS & & & \\
\hline DRB1*11 & 11.93 & 21.9 & 0.0025 & 0.4832 & 0.299 & 0.7806 \\
\hline DRB1*12 & 7.95 & 4.4 & NS & & & \\
\hline DRB1*13 & 22.16 & 20.9 & NS & & & \\
\hline
\end{tabular}




\begin{tabular}{|c|c|c|c|c|c|c|}
\hline \multicolumn{7}{|c|}{ Table 5: Contd... } \\
\hline \multirow[t]{2}{*}{ HLA allele } & \multirow{2}{*}{$\begin{array}{l}\text { Frequencies (\%) in ALL } \\
\qquad(n=176)\end{array}$} & \multirow{2}{*}{$\begin{array}{l}\text { Frequencies }(\%) \text { in control } \\
\qquad(n=1000)\end{array}$} & \multirow[t]{2}{*}{$P$} & \multirow[t]{2}{*}{ OR } & \multicolumn{2}{|c|}{$95 \% \mathrm{CI}$} \\
\hline & & & & & Low & High \\
\hline$\overline{\mathrm{DRB} 1 * 14}$ & 14.20 & 16.1 & NS & & & \\
\hline $\mathrm{DRB} 1 * 15$ & 36.93 & 35.1 & NS & & & \\
\hline $\mathrm{DRB} 1 * 16$ & 1.14 & 2.2 & NS & & & \\
\hline DRB*17 & 0.57 & 23.5 & NS & & & \\
\hline
\end{tabular}

NS - Not significant; HLA - Human leukocyte antigen; OR - Odds ratio; CI - Confidence interval; ALL - Acute lymphoid leukemia

\begin{tabular}{|c|c|c|c|c|c|c|}
\hline \multirow[t]{2}{*}{ HLA allele } & \multirow{2}{*}{$\begin{array}{c}\text { Frequencies (\%) in ALL } \\
(n=164)\end{array}$} & \multirow{2}{*}{$\begin{array}{l}\text { Frequencies }(\%) \text { in control } \\
\qquad(n=1000)\end{array}$} & \multirow[t]{2}{*}{$P$} & \multirow[t]{2}{*}{ OR } & \multicolumn{2}{|c|}{$95 \%$ CI } \\
\hline & & & & & Low & High \\
\hline $\mathrm{C}^{*} 01$ & 9.15 & 7.7 & NS & & & \\
\hline$C^{*} 02$ & 3.05 & 2.6 & NS & & & \\
\hline$C^{*} 03$ & 14.02 & 13.6 & NS & & & \\
\hline$C^{*} 04$ & 24.39 & 28.1 & NS & & & \\
\hline$C^{*} 05$ & 3.66 & 1.9 & NS & & & \\
\hline$C^{*} 06$ & 21.95 & 19.9 & NS & & & \\
\hline$C^{*} 07$ & 45.12 & 43.6 & NS & & & \\
\hline$C^{*} 08$ & 3.66 & 4.7 & NS & & & \\
\hline$C^{*} 12$ & 28.66 & 29.2 & NS & & & \\
\hline$C^{*} 14$ & 3.05 & 6 & NS & & & \\
\hline$C^{*} 15$ & 15.86 & 21 & NS & & & \\
\hline$C^{*} 16$ & 7.32 & 4.5 & NS & & & \\
\hline$C^{*} 17$ & 1.83 & 1.4 & NS & & & \\
\hline$C^{*} 18$ & 0.61 & 0 & NS & & & \\
\hline DQB1*02 & 43.29 & 41.5 & NS & & & \\
\hline $\mathrm{DQB} 1 * 03$ & 40.24 & 45.1 & NS & & & \\
\hline DQB1*04 & 2.44 & 3.8 & NS & & & \\
\hline DQB1*05 & 38.41 & 36.9 & NS & & & \\
\hline $\mathrm{DQB} 1 * 06$ & 47.56 & 45.3 & NS & & & \\
\hline DQB $1 * 15$ & 0.61 & 0 & NS & & & \\
\hline
\end{tabular}

NS - Not significant; HLA - Human leukocyte antigen; OR - Odds ratio; CI - Confidence interval; ALL - Acute lymphoid leukemia

\begin{tabular}{lcc}
\hline \multicolumn{3}{c}{ Table 7: Multivariate analysis results } \\
\hline Outcome & OR $(\mathbf{9 5 \%}$ CI $)$ & $\boldsymbol{P}$ \\
\hline Acute leukemia & $0.719(0.561-0.922)$ & 0.009 \\
A*11 & $6.629(1.725-25.471)$ & 0.006 \\
B*39 & $0.697(0.509-0.955)$ & 0.025 \\
B*51 & $3.949(2.705-5.764)$ & $<0.0001$ \\
DRB1*11 & $8.107(2.135-30.781)$ & \\
AML & $0.489(0.262-0.912)$ & 0.002 \\
B*39 & 0.025 \\
C*01 & $0.617(0.422-0.902)$ & \\
ALL & $2.740(1.593-4.714)$ & 0.013 \\
A*11 & $<0.0001$ \\
DRB1*11 & OR
\end{tabular}

OR - Odds ratio; CI - Confidence interval; ALL - Acute lymphoid leukemia; AML - Acute myeloid leukemia

At HLA-B locus, a statistically significant association between HLA-B*39 $(P<0.05)$ with acute leukemia and with AML was identified. The frequency of HLA-B*39 in patients was higher in acute leukemia and in AML than that of the control population. Earlier, in a study on
Venezuelan population, they also found a strong association between HLA-B*39 and myeloid leukemia using molecular technique. ${ }^{[18]}$ A serology-based study published the HLA-B*39 association with myeloid leukemia. ${ }^{[20]}$ Another study by Klitz et al. reported an increased risk to develop ALL in medical refractory pediatric patients having HLA-B*39. ${ }^{[21]}$ Earlier to this, Fernández-Torres found this allele to be negatively associated with ALL. ${ }^{[22]}$ It will be interesting that high-resolution subtypes of HLA-B*39 are studied to understand its role in leukemia association. There are only few reports of HLA and AML associations made with molecular biology techniques.

Another HLA allele that showed significance in our study was HLA-C*07. It showed the increased frequency in acute leukemia and AML than that in control population. A study found this allele to be overrepresented in chronic lymphoid leukemia population as a whole. ${ }^{[23]}$ In 1988, a study found significant association of ALL with HLA-Cw7 in German population using serology technique. ${ }^{[13]}$ This would indicate that Indians are not only genetically different but also 
possess different and distinct activating factors in leukemia development. ${ }^{[24]}$

\section{Conclusions}

We found significant positive and negative associations with acute leukemia and AML and ALL in this study. Over the years, there are several studies done on HLA associations in different populations; some of the present results support the earlier findings, whereas some are not matching. This is most likely because HLA is highly polymorphic and the frequency of these HLA alleles differs among population. Another reason for this difference could be the sample size difference in all these studies. Many more studies with large sample size on Indian population are required to establish the association of these HLA alleles with the development of acute leukemia.

\section{Acknowledgment}

We are thankful to Gaurav Bhatt, Bhuvanchand, Akhilesh Kumar, Parvind Singh, Richa Chaudhary, Archana Anthwal, Nikki Dey, and Siddhant for their kind support.

\section{Financial support and sponsorship}

Nil.

\section{Conflicts of interest}

There are no conflicts of interest.

\section{References}

1. Hanahan D, Weinberg RA. The hallmarks of cancer. Cell 2000;100:57-70.

2. Pui $\mathrm{CH}$, Relling MV, Downing JR. Acute lymphoblastic leukemia. N Engl J Med 2004;350:1535-48.

3. Terasaki PI, editor. History of HLA: Ten Recollections. Los Angeles: UCLA Tissue Typing Laboratory Press; 1990.

4. Corzo D, Salazar M, Granja CB, Yunis EJ. Advances in HLA genetics. Exp Clin Immunogenet 1995;12:156-70.

5. Baur MP, Danilovs J. Population Genetic Analysis of HLA-A, B, C, DR and other Genetic Markers. In: Terisaki PI, editor. Histocompatibility Testing. Los Angeles (CA): UCLA Tissue Typing Laboratory; 1980. p. 955-93.

6. Ward FE, Amos DB. Human leukocyte antigens and disease. In: Litwin SD, editor. Human Immunogenetics M-Q Basic Principles and Clinical Relevance. New York: Marcel Dekker, Inc.; 1980. p. 359-78.

7. Lechler R, Warrens A. HLA in Health and Disease. London: Academic Press Limited; 2000.

8. Lilly F, Boyse EA, Old LJ. Genetic basis of susceptibility to viral leukemogenesis. Lancet 1964;2:1207-9.

9. Dausset J. The major histocompatibility complex in man. Science 1981;213:1469-74.

10. Walford RL, Finkelstein S, Neerhout R, Konrad P, Shanbrom E. Acute childhood leukemia in relation to the HLA human transplantation genes. Nature 1970;5231:461-2.

11. Seremetis S, Cuttner J, Winchester R. Definition of a possible genetic basis for susceptibility to acute myelogenous leukemia associated with the presence of a polymorphic Ia epitope. J Clin Invest 1985;76:1391-7.

12. Bortin MM, D'Amaro J, Bach FH, Rimm AA, van Rood JJ. HLA associations with leukemia. Blood 1987;70:227-32.

13. Muller CA, Ilasmann R, Grosse Wildee FI. Significant association of acute lymphoblastic leukaemia with HLA-Cw7. Genet Epidemiol 1988;5:453-61.

14. Yoon J. Acute myeloid leukemia is a disease associated with HLA-C3. Acta Haematol 2015;133:164-7.

15. Available from: http://www.genebandhu.in. [Last accessed on 2020 Mar 30].

16. Svejgaard A, Ryder LP. HLA and disease associations: Detecting the strongest association. Tissue Antigens 1994;43:18-27.

17. Fernandes TA, Fukai R, Souza CA, Lorand-Metze I, Magna LA, Kraemer MH. Molecular identification of the HLA-DRB1-DQB1 for diagnosis and follow-up of acute leukemias. Blood Cells Mol Dis 2010;44:69-73.

18. Villalobos C, Rivera S, Weir-Medina J, Hassanhi M, Montiel M, González R. Association of HLA class I and leukemia in mestizo patients of the state of Zulia, Venezuela. Invest Clin 2003;44:283-9.

19. Ozdilli K, Oguz FS, Anak S, Kekik C, Carin M, Gedikoglu G. The frequency of HLA Class I and II alleles in Turkish childhood acute leukaemia patients. J Int Med Res 2010;38:1835-44.

20. Layrisse Z, Guedez Y, Dominguez E, Herrera F, Soto M, Balbas $\mathrm{O}$, et al. Extended HLA haplotypes among the Bari Amerindians of the Perija Range. Relationship to other tribes based on four-loci haplotype frequencies. Hum Immunol 1995;44:228-35.

21. Klitz W, Gragert L, Trachtenberg E. Spectrum of HLA associations: The case of medically refractory pediatric acute lymphoblastic leukemia. Immunogenetics 2012;64:409-19.

22. Fernández-Torres J, Flores-Jiménez D, Arroyo-Pérez A, Granados J, López-Reyes A. HLA-B*40 allele plays a role in the development of acute leukemia in Mexican population: A case-control study. Biomed Res Int 2013;2013:705862.

23. Shah N, Decker WK, Lapushin R, Xing D, Robinson SN, Yang $\mathrm{H}$, et al. HLA homozygosity and haplotype bias among patients with chronic lymphocytic leukemia: Implications for disease control by physiological immune surveillance. Leukemia 2011;25:1036-9.

24. Rothhammer F, Silva C, Callegari-Jacques SM, Llop E, Salzano FM. Gradients of HLA diversity in South American Indians. Ann Hum Biol 1997;24:197-208. 